\title{
LETTER
}

\section{Multidrug-resistant bacterial carriage and related healthcare-associated infections in a pediatric intensive care unit: a 6-year prospective study}

\author{
Michael Levy ${ }^{1,2^{*}}\left(\mathbb{D}\right.$, Stéphane Bonacorsi ${ }^{2,3}$, Jérôme Naudin ${ }^{1,2}$, Marion Caseris ${ }^{2,4}$, Eric Thebault ${ }^{1,2}$, \\ Patricia Mariani-Kurkdjiann ${ }^{2,3}$, Maryline Chomton ${ }^{1,2}$, Julie Sommet ${ }^{1,2}$, Stéphane Dauger ${ }^{1,2}$ and Catherine Doit ${ }^{2,3}$
}

(C) 2019 Springer-Verlag GmbH Germany, part of Springer Nature

Dear Editor,

Few data exist about multidrug-resistant (MDR) bacterial carriage in pediatric intensive care units (PICUs) [1] and its impact on healthcare-associated infections (HCAIs), which cause considerable morbidity and mortality [2]. In adults, a $10 \%$ pooled prevalence of extendedspectrum $\beta$-lactamase-producing Enterobacteriaceae (ESBL-E) colonization at PICU admission has been found, with an additional $7 \%$ acquisition rate during the stay [3]. However, MDR bacterial carriage and its contribution to HCAIs may differ between children and adults because of the lower prevalences of chronic and degenerative organ dysfunction in the former. Our objectives were to estimate the 6-year prevalence of MDR bacterial carriage and to describe the epidemiology of related HCAIs in our PICU.

This single-center prospective cohort study performed at the Robert-Debre University Hospital (Paris, France) included consecutive children admitted to a tertiary 20-bed PICU in 2011-2016. Rectal and nasal swabs were collected routinely at admission and weekly thereafter to detect MDR bacteria (ESBL-E and methicillinresistant Staphylococcus aureus, MRSA). MDR bacterial carriage was defined as imported when the admission swab was positive or when carriage was identified before

\footnotetext{
*Correspondence: levy-michael@live.fr

${ }^{1}$ Pediatric Intensive Care Unit, Robert-Debré University Hospital,

Assistance Publique-Hôpitaux de Paris, 48, boulevard Sérurier, 75019 Paris, France

Full author information is available at the end of the article
}

admission, and as acquired when the admission swab was negative and a subsequent swab positive. We recorded all major HCAIs including ventilator-associated pneumonia (VAP), central line-associated bloodstream infections (CLABSI), and catheter-associated urinary tract infections (CAUTI), defined on the basis of Centers for Disease Control criteria and confirmed retrospectively [4].

Of 5419 patients admitted during the study period (Supplementary Table 1), 550 (10.1\%) carried ESBL$\mathrm{E}$ and 99 (1.8\%) MRSA. The ESBL-E incidence rate increased from 12.8 to $23.0 / 1000$ patient-days as a result of an increase in imported cases $(P=0.13)$, contrasting with a steady decrease in acquired cases $(P=0.06)$ with an acquisition rate of only $1 \%$. Likewise, the MRSA carriage incidence rate rose over time from 2.04 to $5.56 / 1000$ patient-days as a result of an increase in imported MRSA cases $(P=0.28)$ and despite a decrease in acquired cases $(P=0.14)$ (Fig. 1). Over time, use declined for third-generation cephalosporins $(P=0.04)$ and increased for penicillins (mainly amoxicillin); carbapenem and vancomycin use tended to decline $(P=0.59$ and 0.07 , respectively) (Supplementary Fig. 1). The annual HCAI prevalence ranged from $1.9 \%$ to $4.3 \%$ and the incidence rate from 3.3 to $6.2 / 1000$ patient-days. The prevalence of HCAIs due to MRSA, ESBL E. coli, and ESBL $K$. pneumoniae among carriers was 3\% (3/99), $1.7 \%(6 / 350)$, and $3.0 \%(5 / 165)$, respectively. The 146 major HCAIs comprised $41.8 \%$ VAPs, $39 \%$ CLABSIs, and 19.2\% CAUTIs. Of all these HCAIs, 13 (8.9\%) were due to ESBL-E and $3(2.0 \%)$ to MRSA, all in previously identified carriers. 

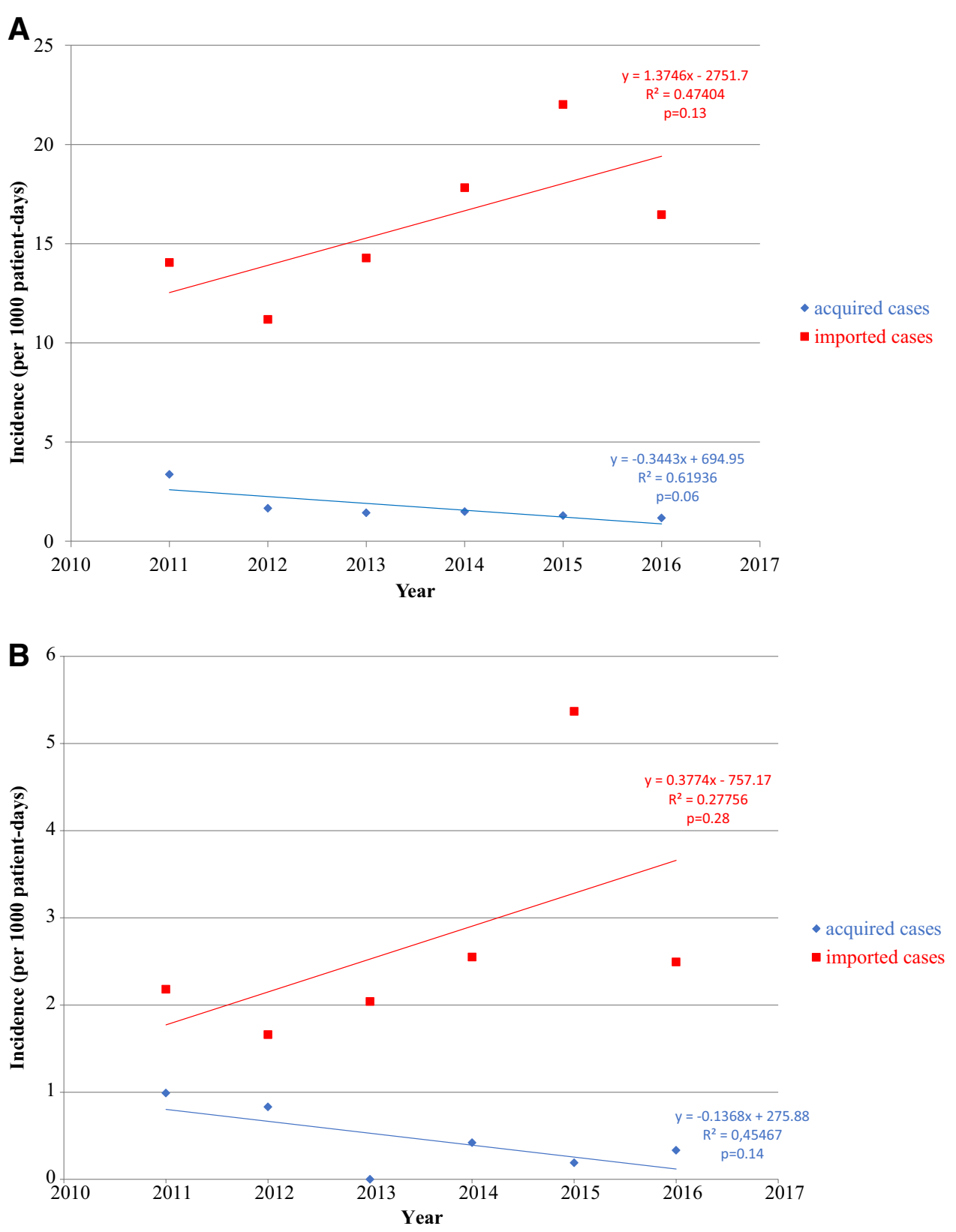

Fig. 1 Incidence rate of extended-spectrum $\beta$-lactamase Enterobacteriaceae (a) and methicillin-resistant Staphylococcus aureus (b) carriage per 1000 patient-days

Despite a steady increase in MDR bacteria carriers at admission over the 6-year period, HCAIs due to carried MDR bacteria were rare in our PICU, suggesting that preventive measures were effective. All MDR-HCAIs (i.e., due to ESBL-E or MRSA) occurred in patients previously identified by routine surveillance swabs as carriers of the culprit organism. Therefore, incorporating routine screening results into first-line antibiotic selection, combined with effective preventive measures against MDR bacteria transmission, may decrease the inappropriate use of vancomycin and carbapenems, as suggested by data from adult ICUs [5].

\section{Electronic supplementary material}

The online version of this article (https://doi.org/10.1007/s00134-019-05584-3) contains supplementary material, which is available to authorized users. 


\section{Author details}

${ }^{1}$ Pediatric Intensive Care Unit, Robert-Debré University Hospital, Assistance Publique-Hôpitaux de Paris, 48, boulevard Sérurier, 75019 Paris, France. ${ }^{2}$ University Paris Diderot Sorbonne Paris Cité, Paris, France. ${ }^{3}$ Microbiology Unit, Robert-Debré University Hospital, Assistance Publique-Hôpitaux de Paris, Paris, France. ${ }^{4}$ Pediatric Infectious Disease Unit, Robert-Debré University Hospital, Assistance Publique-Hôpitaux de Paris, Paris, France.

\section{Compliance with ethical standards}

\section{Conflicts of interest}

No conflict of interests to declare.

\section{Ethical approval}

The study was approved by the International Review Board of Robert-Debré Hospital, Paris, France (No 2018-422).

\section{Publisher's Note}

Springer Nature remains neutral with regard to jurisdictional claims in published maps and institutional affiliations.

Accepted: 25 February 2019

Published online: 6 March 2019

\section{References}

1. Suwantarat N, Logan LK, Carroll KC et al (2016) The prevalence and molecular epidemiology of multidrug-resistant Enterobacteriaceae colonization in a pediatric intensive care unit. Infect Control Hosp Epidemiol 37:535-543. https://doi.org/10.1017/ice.2016.16

2. Alten JA, Rahman AKMF, Zaccagni HJ et al (2018) The epidemiology of healthcare-associated infections in pediatric cardiac intensive care units. Pediatr Infect Dis J 37:768-772. https://doi.org/10.1097/INF.0000000000 001884

3. Detsis M, Karanika S, Mylonakis E (2017) ICU acquisition rate, risk factors, and clinical significance of digestive tract colonization with extendedspectrum beta-lactamase-producing Enterobacteriaceae: a systematic review and meta-analysis. Crit Care Med 45:705-714. https://doi. org/10.1097/CCM.0000000000002253

4. The Centers for Disease Control and Prevention (2016) National Healthcare Safety Network Patient Safety Component Manual. CDC, Atlanta

5. Carbonne H, Le Dorze M, Bourrel A-S et al (2017) Relation between presence of extended-spectrum $\beta$-lactamase-producing Enterobacteriaceae in systematic rectal swabs and respiratory tract specimens in ICU patients. Ann Intensive Care 7:13. https://doi.org/10.1186/s13613-017-0237-x 